\title{
ON MULTIPLICATION TABLES OF GROUPS THAT AGREE ON HALF OF THE COLUMNS AND HALF OF THE ROWS
}

\author{
ALEŠ DRÁPAL and NATALIA ZHUKAVETS* \\ Department of Mathematics and Physics (MFF), Karlova (Charles) University (UK), 18675 Prague 8 , \\ Czech Republic \\ e-mail:drapal@karlin.mff.cuni.cz
}

(Received 11 March, 2002; accepted 14 November, 2002)

\begin{abstract}
Let $G(\circ)$ and $G(*)$ be two groups of finite order $n$, and suppose that each of the sets $\{u \in G ; u \circ v=u * v$ for all $v \in G\}$ and $\{v \in G ; u \circ v=u * v$ for all $u \in G\}$ has $n / 2$ elements. Then $G(*)$ can be obtained from $G(\circ)$ by one of the two general constructions that are discussed in the paper.
\end{abstract}

2000 Mathematics Subject Classification. 20D60, 05B15.

For finite groups $G(\circ)$ and $G(*)$ denote the size of $\{(u, v) \in G \times G ; u \circ v \neq u * v\}$ by $d(\circ, *)$. If $n$ is a power of two, $n=|G|$, and $d(\circ, *)<n^{2} / 4$, then $G(\circ) \cong G(*)$, by [3]. Hence 2-groups $G(\circ)$ and $G(*)$ with $d(\circ, *)=n^{2} / 4$ deserve a special attention. It is not difficult to find examples of such groups $[\mathbf{1}, \mathbf{2}, \mathbf{3}]$. Natalia Zhukavets systematically studied the case $n \leq 32$ in her thesis [7], and in this context she and the first author discovered two general constructions of this paper that are responsible for the majority of pairs $(G(\circ), G(*))$ with $d(\circ, *)=n^{2} / 4$, if $n=|G|$ is a power of two and $n \leq 64$.

Put $U=\{u \in G ; u \circ v=u * v$ for all $v \in G\}$ and $V=\{v \in G ; u \circ v=u * v$ for all $u \in G$ \}. The sets $U$ and $V$ can be, in general, empty. However, if $U$ (or $V$ ) is non-empty, then it is a subgroup of both $G(\circ)$ and $G(*)$. This can be verified easily and we shall show that both $U$ and $V$ are non-empty if and only if $G(\circ)$ and $G(*)$ share the unit element.

If $|G: U|=2=|G: V|$, then clearly $d(\circ, *) \leq n^{2} / 4$. However, the case $d(\circ, *)<$ $n^{2} / 4$ never occurs, and it turns out that conditions $d(\circ, *)=n^{2} / 4$ and $|G: U|=2=$ $|G: V|$ are strong enough to enable a rather detailed description of the relationship of $G(\circ)$ and $G(*)$. This description can be turned into a prescription of how one can obtain, given a group $G(\circ)$, all groups $G(*)$ with $|G: U|=2=|G: V|$ such that $x \circ y=x * y$ if and only if $x \in U$ or $y \in V$. This prescription has the form of two constructions, one corresponding to the case $U=V$ and the other one to the case $U \neq V$.

Basic properties of these constructions and their characterizations are the content of Sections 1 and 2. In the second part of Section 1 we also give some auxiliary results that are used in Sections 3 and 4 when determining the cases, in which the constructions yield a group that is isomorphic to the original group. Theorems 3.4 and 3.8 give a more abstract description of situations when a group can be obtained from another group by one of our constructions, and Section 5 deals with groups that can be obtained in

\footnotetext{
*Work supported by institutional grant MSM 113200007 and by Grant Agency of Charles Univ., grant number 269/2001/B-MAT/MFF.
} 
this way from finite abelian groups. Related papers and some prospects for future work are mentioned at the end of the paper.

The main advantage of the presented constructions seems to be their simplicity. One could easily interpret them in the language of group extensions, or in other ways. However, we have refrained from doing so, since it is not clear how such an interpretation might prove useful with respect to our aims. Our goal is to find a system of simple passages between 2-groups of the same order, where any two groups of that order are (transitively) connected. This could lead to a new approach to 2-groups, when a property might be established by a verification for a single group and by a proof that it is retained by each passage. The methods of this paper make interconnected all 2 -groups of order $n$ when $n \leq 16$. A slight generalization yields the case $n=32$, and there are reasons to hope that other kinds of passages will be found for higher orders.

When dealing with operations $\circ$ and $*$, we denote the unit elements by $1_{\circ}$ and $1_{*}$, and the inverse elements by $x^{-1}$ and $x^{*}$, respectively.

1. Agents of disagreement. Suppose that $G(\circ)$ and $G(*)$ are groups of a finite order $n$ with $d(\circ, *)=n^{2} / 4$. The set $\left\{(x \circ y)^{-1} \circ(x * y) ; x, y \in G\right\}$ certainly contains 1 , and we shall see that under certain additional conditions this set contains, besides the unit element, either just one element, or two elements that are mutually inverse.

Lemma 1.1. Let $\circ$ and $*$ be two group operations on the set $G$. Put $U=\{u \in$ $G ; u \circ v=u * v$ for all $v \in G\}$ and $V=\{v \in G ; u \circ v=u * v$ for all $u \in G\}$. Then either both $U$ and $V$ are empty, or $\circ$ and $*$ share the unit element. In the latter case both $U$ and $V$ are subgroups of $G(\circ)$ and of $G(*)$.

Proof. If there is a common unit, then it is in $U \cap V$. Conversely, if $u \in U$, then $u \circ 1_{\circ}=u=u * 1_{*}=u \circ 1_{*}$, and $1_{\circ}=1_{*}$. Hence we can assume, for the rest of the proof, that $\circ$ and $*$ share the unit element 1 . Recall that by $x^{*}$ we denote the inverse of $x \in G$ with respect to $*$. For $u \in U$ one has $u \circ u^{-1}=1=u * u^{*}=u \circ u^{*}$, and $u^{-1}=u^{*}$ follows. For every $y \in G$ there is $u \circ\left(u^{-1} \circ y\right)=y=u *\left(u^{-1} * y\right)=u \circ\left(u^{-1} * y\right)$, which means $u^{-1} \circ y=u^{-1} * y$ and $u^{-1} \in U$. If $u_{1}, u_{2} \in U$ and $y \in G$, then $\left(u_{1} \circ u_{2}\right) \circ y=$ $u_{1} \circ\left(u_{2} \circ y\right)=u_{1} *\left(u_{2} * y\right)=\left(u_{1} * u_{2}\right) * y=\left(u_{1} \circ u_{2}\right) * y$, and the rest is clear.

THEOREM 1.2. Let $\circ$ and $*$ be two different group operations on a set $G$, and suppose that each of the subgroups $U=\{u \in G ; u \circ v=u * v$ for all $v \in G\}$ and $V=\{v \in G ; u \circ$ $v=u * v$ for all $u \in G\}$ is of index 2 in both $G(\circ)$ and $G(*)$. Put $S=U \cap V$.

If $U=V=S$, then there exists a (unique) $h \in S \cap Z(G(\circ))$ such that for all $x, y \in G$

$$
x * y= \begin{cases}x \circ y, & \text { if } x \in S \text { or } y \in S \\ x \circ y \circ h, & \text { if } x \in G \backslash S \text { and } y \in G \backslash S .\end{cases}
$$

If $U \neq V$, then there exists a (unique) $h \in Z(S)$ with $h \circ u \circ h=u$ for all $u \in U \backslash S$ and $h \circ v \circ h=v$ for all $v \in V \backslash S$ such that for all $x, y \in G$

$$
x * y= \begin{cases}x \circ y, & \text { if } x \in U \text { or } y \in V, \\ x \circ y \circ h, & \text { if } x \in G \backslash U \text { and } y \in U \backslash V, \\ x \circ y \circ h^{-1}, & \text { if } x \in G \backslash U \text { and } y \in G \backslash(U \cup V) .\end{cases}
$$


Proof. Suppose first $U=V$. If $x \in S$ or $y \in S$, then $x \circ y=x * y$, by the very definition of $U$ and $V$. Consider $x, y \in G \backslash S$ with $x \circ y \neq x * y$. Then for all $u, v \in$ $G \backslash S$ there exist $s, t \in S$ such that $v=s \circ y=s * y$ and $u=t \circ x \circ s^{-1}=t * x * s^{-1}$. This means $u \circ v \neq u * v$, as $u \circ v=t \circ x \circ y=t *(x \circ y)$ and $u * v=t *(x * y)$. Put $h=(x \circ y)^{-1} \circ(x * y)$, and note that $(u \circ v)^{-1} \circ(u * v)$ is also equal to (the "agent") $h$. Hence $u * v=u \circ v \circ h$ for all $u, v \in G \backslash S$, and it remains to show $h \in Z(G(\circ))$. However, for every $u \in G \backslash S$ we have $u * u * u=u *(u \circ u \circ h)=(u \circ u) \circ(u \circ h)$ and $u * u * u=(u \circ u \circ h) * u=(u \circ u) \circ(h \circ u)$.

Assume now $U \neq V$. Then $G(\circ) / S \cong G(*) / S$ is isomorphic to Klein's 4-group, and $W=S \cup(G \backslash(U \cup V))$ is a subgroup of both $G(\circ)$ and $G(*)$. Put $U_{1}=U \backslash S$, $V_{1}=V \backslash S$ and $W_{1}=W \backslash S$. If $x \in G \backslash U=V_{1} \cup W_{1}$, then there exists $y \in G \backslash V=$ $U_{1} \cup W_{1}$ with $x \circ y \neq x * y$. Suppose first $y \in U_{1}$. Every $z \in W_{1}$ can be expressed as $y \circ v, v \in V_{1}$, and $x \circ z=x \circ y \circ v=(x \circ y) * v \neq(x * y) * v=x * z$. By reversing this procedure one derives $x \circ y \neq x * y$ for all $y \in U_{1}$, if $x \circ z \neq x * z$ for some $z \in W_{1}$. Hence $x \circ y \neq x * y$ for $x, y \in G$ if and only if $x \in G \backslash U=V_{1} \cup W_{1}$ and $y \in G \backslash V=$ $U_{1} \cup W_{1}$, and so for $x, y \in W$ one gets $x \circ y \neq x * y$ just when $x, y \in W_{1}$. By the first part of the proof there exists $h_{1} \in S \cap Z(W(\circ))$ with $x * y=x \circ y \circ h_{1}$ for all $x, y \in W_{1}$. Put $h=h_{1}^{-1}$.

If $x, y \in W_{1}$ and $u \in U_{1}$, then $(u \circ x) * y=u * x * y=u *\left(x \circ y \circ h^{-1}\right)=((u \circ x) \circ$ $y) \circ h^{-1}$, and so one obtains $x^{\prime} * y=x^{\prime} \circ y \circ h^{-1}$ for all $\left(x^{\prime}, y\right) \in\left(V_{1} \cup W_{1}\right) \times W_{1}$.

Consider now $u \in U_{1}$ and $v \in V_{1}$, and put $x=u^{-1} \circ v^{-1}=u^{-1} * v^{-1}=u^{*} * v^{*}$. From $1=x * x^{*}=x \circ x^{*} \circ h^{-1}$ we get $x^{*}=x^{-1} \circ h$, and $v * u=x^{*}=x^{-1} \circ h=v \circ$ $u \circ h$ follows. If $u^{\prime}$ is a further element of $U_{1}$, then $\left(u^{\prime} \circ v\right) * u=u^{\prime} * v * u=u^{\prime} *(v \circ u \circ$ $h)=\left(\left(u^{\prime} \circ v\right) \circ u\right) \circ h$, and hence $x * y=x \circ y \circ h$ for all $(x, y) \in\left(V_{1} \cup W_{1}\right) \times U_{1}$.

Now, for $u \in U_{1}$ and $v \in V_{1}$ we also obtain $v \circ u \circ h \circ u \circ h=(v \circ u \circ h) * u=$ $(v * u) * u=v *(u * u)=v \circ u \circ u$, and $h \circ u \circ h=u$ follows. Therefore $u \circ h \circ v \circ h=$ $h^{-1} \circ u \circ v \circ h=h^{-1} \circ h \circ u \circ v=u \circ v$, and $h \circ v \circ h=v$.

Theorem 1.2 was obtained by generalizing examples of 2-groups of small order $n$ with $d(\circ, *)=n^{2} / 4$. Nevertheless, there is no need to assume that $G$ is finite in the theorem: it suffices to require $|G: U|=2=|G: V|$.

In the rest of this section we shall make several observations that mainly pertain to conditions of the form $h u h=u$ and $h v h=v$. These observations will be made in the context of just one group operation.

For a subset $M$ of a group $G$ denote by $\langle M\rangle$ the least subgroup of $G$ that contains $M$. Note that $\langle G \backslash T\rangle=G$ when $T<G$ is of index 2 . For every $M \subseteq G$ put

$$
Q(M)=\{h \in\langle M\rangle \backslash M ; h g h=g \text { for all } g \in M\} .
$$

Proposition 1.3. Assume $T<G$ and $|G: T|=2$. Then $Q(G \backslash T) \leq Z(T)$, and $h \in Z(T)$ belongs to $Q(G \backslash T)$ if and only if $h g h=g$ for some (and thus for all) $g \in G \backslash T$.

Proof. Each $t \in T$ can be expressed as $g_{1} g_{2}$, where $g_{1}, g_{2} \in G \backslash T$. If $h \in Q(G \backslash T)$, then $h t h^{-1}=h g_{1} g_{2} h^{-1}=h g_{1} h h^{-1} g_{2} h^{-1}=g_{1} g_{2}=t$. Hence $Q(G \backslash T) \subseteq Z(T)$, and for $h_{1}, h_{2} \in Q(G \backslash T)$ one gets $h_{1} h_{2}=h_{2} h_{1}$ and $h_{1} h_{2} g h_{1} h_{2}=h_{1} h_{2} g h_{2} h_{1}=h_{1} g h_{1}=g$, for all $g \in G \backslash T$. If $h \in Q(G \backslash T)$ then clearly $h^{-1} \in Q(G \backslash T)$, and so $Q(G \backslash T)$ really forms a subgroup of $Z(T)$.

Suppose now $h g h=g$ for some $g \in G \backslash T$, where $h \in Z(T)$. Then $h g t h=$ $h g h h^{-1} t h=g t$ for all $t \in T$, and hence $h \in Q(G \backslash T)$. 
For a group $G$ and its subsets $M_{1}$ and $M_{2}$ put

$$
Q\left(M_{1}, M_{2}\right)=Q\left(M_{1} \backslash M_{2}\right) \cap Q\left(M_{2} \backslash M_{1}\right) .
$$

To indicate which group operation is used we write, e.g., $Q^{\circ}(M)$ or $Q^{*}\left(M_{1}, M_{2}\right)$.

Note that $h \in Q^{\circ}(U, V)$ in the situation of Theorem 1.2 if $U \neq V$. In such a case we clearly have $Q^{\circ}(U, V)=Q^{*}(U, V)$, and hence we write just $Q(U, V)$. This set consists of all $h \in S$ with $h \circ x \circ h=x$ for every $x \in G \backslash W$, where $S<W<$ $G$ and $(W \backslash S)=(U \backslash S) \circ(V \backslash S)=(U \backslash S) *(V \backslash S)$. Therefore $Q(U, V)=S \cap$ $Q^{\circ}(G \backslash W)$ and $Q^{\circ}(G \backslash W) \leq Z(W(\circ))$, by Proposition 1.3.

Lemma 1.4. Assume $h \in Q(G \backslash T)$, where $T<G$ and $|G: T|=2$. Set $\alpha_{h}(t)=t$ for $t \in T$ and $\alpha_{h}(g)=$ gh for $g \in G \backslash T$. Then $\alpha_{h} \in \operatorname{Aut}(G)$ and each $\alpha \in \operatorname{Aut}(G)$ that fixes $T$ pointwise is of such a form.

Proof. Fix $g \in G \backslash T$ and $t \in T$, and note that $h \in Z(T)$, by Proposition 1.3. We have $\alpha_{h}(g t)=g t h=g h t=\alpha_{h}(g) \alpha_{h}(t), \alpha_{h}(t g)=t g h=\alpha_{h}(t) \alpha_{h}(g)$ and $\alpha_{h}(g \cdot g t)=$ $g^{2} t=g h g t h=\alpha_{h}(g) \alpha_{h}(g t)$. On the other hand, if $\alpha \in \operatorname{Aut}(G)$ fixes $T$ pointwise, set $h=g^{-1} \alpha(g)$. Then $\alpha(t g)=t \alpha(g)=t \cdot g h$, and $g h g h=\alpha(g) \alpha(g)=\alpha\left(g^{2}\right)=g \cdot g$ yields $h \in Q(G \backslash T)$.

Lemma 1.5. Assume $h \in Q(G \backslash T)$, where $T<G$ and $|G: T|=2$. Then $h^{g}=h^{-1}$ for every $g \in G \backslash T$.

Proof. The equality $g^{-1} h g=h^{-1}$ is just another form of the equality $h g h=g$.

Lemma 1.6. Assume $z \in Z(T)$, where $T<G$ and $|G: T|=2$. Then $z^{g^{\prime}} z=z^{g} z \in$ $Z(G)$ for all $g, g^{\prime} \in G \backslash T$.

Proof. Consider $g, g^{\prime} \in G \backslash T$. There exists $t \in T$ with $g^{\prime}=t g$, and $z^{g^{\prime}}=z^{t g}=z^{g}$. The element $z^{g} z$ belongs to $Z(T)$, and it remains to show $\left(z^{g} z\right)^{g}=z^{g} z$. However, $\left(z^{g} z\right)^{g}=g^{-2} z g z g=z g^{-2} g z g=\left(g^{-1} z g\right) z=z^{g} z$.

LeMmA 1.7. Let $U$ and $V$ be two distinct subgroups of $G$ with $|G: U|=2=\mid G$ : $V \mid$. Set $S=U \cap V$ and denote by $W$ the subgroup of $G$ with $|G: W|=2, S<W$, and $W / S=U / S \cdot V / S$. Then $Q(U, V) \leq Q(U \backslash S) Q(V \backslash S) \leq Z(S)$, and for $p \in Q(U \backslash$ $S), q \in Q(V \backslash S)$ there is $p^{u}=p^{-1}$ for all $u \in U \backslash S$ and $q^{v}=q^{-1}$ for all $v \in V \backslash S$. Furthermore, $p^{v}=p^{v^{\prime}} \in Q(U \backslash S)$ for all $v, v^{\prime} \in V \backslash S$ and $q^{u}=q^{u^{\prime}} \in Q(V \backslash S)$ for all $u, u^{\prime} \in U \backslash S$. Set $z=p^{v} q$ and $w=u v$. Then $z^{w} z=z^{w^{\prime}} z \in Q(U, V)$ for all $w^{\prime} \in W \backslash S$, and $z^{w}=\left(q^{u} p\right)^{-1}$.

Proof. We have $Q(U \backslash S) \leq Z(S)$ and $Q(V \backslash S) \leq Z(S)$, by Proposition 1.3, and $Q(U, V)=Q(U \backslash S) \cap Q(V \backslash S)$, by the definition. Thus $Q(U, V) \leq Q(U \backslash S) Q(V \backslash$ $S) \leq Z(S)$.

Fix $p \in Q(U \backslash S)$ and $q \in Q(V \backslash S)$. Then $p^{u}=p^{-1}$ and $q^{v}=q^{-1}$, by Lemma 1.5. Relations $p^{v}=p^{v^{\prime}}$ and $q^{u}=q^{u^{\prime}}$ follow from $p, q \in Z(S)$. From $(V \backslash S)^{u}=V \backslash S$ and $(U \backslash S)^{v}=U \backslash S$ we get $p^{v} \in Q(U \backslash S)$ and $q^{u} \in Q(V \backslash S)$. Hence $z=p^{v} q \in Z(S)$, and $z^{w} z=z^{w^{\prime}} z \in Z(W)$ follows from Lemma 1.6. We have $w=u v$ and $z^{w}=\left(p^{v} q\right)^{u v}=$ $\left(\left(p^{v}\right)^{-1} q^{u}\right)^{v}=\left(p^{v^{2}}\right)^{-1}\left(q^{u}\right)^{-1}=\left(q^{u} p\right)^{-1}$. Recall that all of $p, q, p^{v}$ and $q^{u}$ belong to $Z(S)$. Hence

$$
\begin{aligned}
z^{w} z u z^{w} z & =p^{-1}\left(q^{u}\right)^{-1} p^{v} q u p^{-1}\left(q^{u}\right)^{-1} p^{v} q=\left(q^{u}\right)^{-1} q p^{-1} p^{v} u p^{v} p^{-1}\left(q^{u}\right)^{-1} q \\
& =\left(q^{u}\right)^{-1} q u\left(q^{u}\right)^{-1} q=\left(q^{u}\right)^{-1} u q=u^{-1} q^{-1} u^{2} q=u^{-1} u^{2} q^{-1} q=u,
\end{aligned}
$$

and, similarly, $z^{w} z v z^{w} z=p^{-1} p^{v} v p^{-1} p^{v}=p^{-1} v^{-1} p v^{2} p^{-1} v^{-1} p v=v$, as $p v^{2}=v^{2} p$. 
2. Constructions. We shall now show that conditions on $S, U, V$ and $h$, as expressed in Theorem 1.2, suffice to construct new group operations.

Proposition 2.1. Let $G=G(\cdot)$ be a group, $S<G$ a subgroup of index 2, and $h \in S \cap Z(G)$. Define a new operation $*$ on $G$ by

$$
x * y= \begin{cases}x y, & \text { if } x \in S \text { or } y \in S, \\ x y h, & \text { if } x \in G \backslash S \text { and } y \in G \backslash S .\end{cases}
$$

Then $G(*)$ is a group.

Proof. We have $x * y=x y h^{\pi(x) \pi(y)}$, where $\pi: G \rightarrow\{0,1\}$ maps $S$ to 0 , and $G \backslash S$ to 1 . Denote by $\oplus$ the addition on $\{0,1\}$ modulo 2 , and put $\varepsilon_{i}=\pi\left(g_{i}\right)$, where $g_{i} \in G, 1 \leq$ $i \leq 3$. Then $\left(g_{1} * g_{2}\right) * g_{3}=\left(g_{1} g_{2} h^{\varepsilon_{1} \varepsilon_{2}}\right) * g_{3}=\left(\left(g_{1} g_{2}\right) * g_{3}\right) h^{\varepsilon_{1} \varepsilon_{2}}=g_{1} g_{2} g_{3} h^{\left(\varepsilon_{1} \oplus \varepsilon_{2}\right) \varepsilon_{3}+\varepsilon_{1} \varepsilon_{2}}$ and $g_{1} *\left(g_{2} * g_{3}\right)=g_{1} *\left(g_{2} g_{3} h^{\varepsilon_{2} \varepsilon_{3}}\right)=g_{1} g_{2} g_{3} h^{\varepsilon_{1}\left(\varepsilon_{2} \oplus \varepsilon_{3}\right)+\varepsilon_{2} \varepsilon_{3}}$. The equality $\left(\varepsilon_{1} \oplus \varepsilon_{2}\right) \varepsilon_{3}+$ $\varepsilon_{1} \varepsilon_{2}=\varepsilon_{1}\left(\varepsilon_{2} \oplus \varepsilon_{3}\right)+\varepsilon_{2} \varepsilon_{3}$ is clear when $\varepsilon_{1}=\varepsilon_{2}=\varepsilon_{3}=1$, and can be easily verified when some $\varepsilon_{i}, 1 \leq i \leq 3$, is equal to 0 .

Proposition 2.2. Let $G=G(\cdot)$ be a group, $U<G$ and $V<G$ subgroups of index 2, $U \neq V$, and let $h \in S, S=U \cap V$, be such that huh $=u$ for all $u \in U \backslash S$ and $h v h=v$ for all $v \in V \backslash S$. Define a new operation $*$ on $G$ by

$$
x * y= \begin{cases}x y, & \text { if } x \in U \text { or } y \in V, \\ x y h, & \text { if } x \notin U \text { and } y \in U \backslash V, \\ x y h^{-1}, & \text { if } x \notin U \text { and } y \in G \backslash(U \cup V) .\end{cases}
$$

Then $G(*)$ is a group.

Proof. We can assume $h \neq 1$. For every $x, y \in G$ there exists $\varepsilon \in\{-1,0,1\}$ such that $x * y=x y h^{\varepsilon}$. If $\varepsilon \neq 0$, then the value of $\varepsilon$ depends only on $y$. If $u \in U$, then $u x=u * x$ falls into $U$ just when $x \in U$, and hence $u *(x * y)=u x y h^{\varepsilon}=(u x) * y=(u * x) * y$ for all $x, y \in G$.

The definition of $*$ is left-right symmetric, as it can be expressed by

$$
x * y= \begin{cases}x y, & \text { if } y \in V \text { or } x \in U, \\ h x y, & \text { if } y \notin V \text { and } x \in V \backslash U, \\ h^{-1} x y, & \text { if } y \notin V \text { and } x \in G \backslash(U \cup V) .\end{cases}
$$

For the proof of the above formula put $W=S \cup(G \backslash(U \cup V))$ and note that Proposition 1.3 yields $h \in Z(W)$, as we assume $h x h=x$ for all $x \in G \backslash W$. The formula now follows by considering the four cases with $x \in(V \backslash S) \cup(W \backslash S)$ and $y \in(U \backslash S) \cup(W \backslash S)$. For example, if $x \in W \backslash S$ and $y \in U \backslash S$, then $x * y=x y h=$ $x h^{-1} y=h^{-1} x y$.

The left-right symmetry gives $(x * y) * v=x *(y * v)$ for all $x, y \in G$ and $v \in V$. If $(x * y) * z=x *(y * z)$ and $u \in U$, then $((u * x) * y) * z=(u *(x * y)) * z=u *((x *$ $y) * z)=u *(x *(y * z))=(u * x) *(y * z)$, and, similarly, $(x * y) *(z * v)=x *(y *$ $(z * v))$, provided $v \in V$. We see that it suffices to verify $(x * y) * z=x *(y * z)$ just for $x, z \in W \backslash S$. The case $y \in W$ follows from Proposition 2.1, and the left-right symmetry allows us to assume $y \in U \backslash V$. Then $(x * y) * z=(x y h) * z=x y h z h^{-1}=x y z$ and $x *(y * z)=x *(y z)=x y z$.

The associative law has been verified and the rest is easy. 
The group $G(*)$ of Proposition 2.1 will be denoted by $G[S, h]$, while the group $G(*)$ of Proposition 2.2 will be denoted by $G[U, V, h]$.

By writing $G(*)=G[S, h]$ we shall mean that $G=G(\cdot)$ is a group, $S<G$ its subgroup of index 2 and $h \in S \cap Z(G)$.

Similarly, $G(*)=G[U, V, h]$ means that $U$ and $V$ are distinct subgroups of $G=G(\cdot)$, with $|G: U|=|G: V|=2$, and that $h \in U \cap V$ belongs to $Q(U, V)$. In such a situation we shall denote $U \cap V$ always by $S$, and $S \cup(G \backslash(U \cup V))$ by $W$. If $G(*)=G[U, V, h]$, then $W(*)=W\left[S, h^{-1}\right]$ and $Q(U, V) \leq Q(G \backslash W) \leq Z(W)$, by Proposition 1.3. These facts will be used below without reference.

If $G(*)$ and $G(\cdot)$ are distinct finite groups, where the multiplication tables agree on the half of the columns and the half of the rows, then either $G(*)=G[S, h]$ for some $S$ and $h \neq 1$, or $G(*)=G[U, V, h]$ for some $U, V$ and $h \neq 1$, by Theorem 1.2.

We shall observe that $G(*)$ shares with $G=G(\cdot)$ a large part of its subgroup structure. This also holds for normal subgroups, and to this purpose we first compare conjugation in both groups.

Proposition 2.3. Consider a group $G=G(\cdot)$. If $G(*)=G[S, h]$, then $x^{*}=x^{-1}$ if $x \in S, x^{*}=x^{-1} h^{-1}$ if $x \in G \backslash S$, and $x y x^{-1}=x * y * x^{*}$ for all $x, y \in G$.

If $G(*)=G[U, V, h]$, then $x^{*}=x^{-1}$ if $x \in U \cup V, x^{*}=x^{-1} h$ if $x \in W \backslash S$, and

$$
x * y * x^{*}= \begin{cases}x y x^{-1}, & \text { when }\{x, y\} \subseteq U \text { or }\{x, y\} \subseteq V \text { or }\{x, y\} \subseteq W \\ x y x^{-1} h^{-1}, & \text { when } x \in V \backslash S \text { and } y \in U \backslash S \\ x y x^{-1} h, & \text { in all other cases }\end{cases}
$$

Proof. Assume first $G(*)=G[S, h]$. Suppose $y \in G \backslash S$. If $x \in S$, then $x^{*}=x^{-1}$ and $x * y * x^{*}=(x y) * x^{-1}=x y x^{-1}$, while for $x \in G \backslash S$ we get $x^{*}=x^{-1} h^{-1}$, as $x *\left(x^{-1} h^{-1}\right)=x x^{-1} h^{-1} h=1$, and $x * y * x^{*}=(x y h) *\left(x^{-1} h^{-1}\right)=$ $x y h x^{-1} h^{-1}=x y x^{-1}$. Every $y \in S$ can be expressed as $y_{1} * y_{2}$, where $y_{1}, y_{2} \in G \backslash S$, and $\quad x * y * x^{*}=\left(x * y_{1} * x^{*}\right) *\left(x * y_{2} * x^{*}\right)=\left(x y_{1} x^{-1}\right) *\left(x y_{2} x^{-1}\right)=x y_{1} y_{2} x^{-1} h=$ $x y_{1} y_{2} h x^{-1}=x\left(y_{1} * y_{2}\right) x^{-1}=x y x^{-1}$, for every $x \in G$.

Assume now $G(*)=G[U, V, h]$. Then $U(*)=U(\cdot), V(*)=V(\cdot)$ and for $W(*)=$ $W\left[S, h^{-1}\right]$ the first part of the proof can be used. It remains to express $x * y * x^{*}$ when $x$ and $y$ do not belong together to $U$ or $V$ or $W$, respectively. This means, in particular, that neither $x \in S$, nor $y \in S$. If $x \in U \backslash S$, then $x * y * x^{*}=(x y) * x^{-1}$ is equal to $x y x^{-1} h$ since $x y \notin U$. If $x \in V \backslash S$, then $x * y * x^{*}=x *\left(y x^{-1}\right)=x y x^{-1} h^{ \pm 1}$, where $y x^{-1} \in U \backslash S$ for $y \in W \backslash S$, while $y x^{-1} \in W \backslash S$ for $y \in U \backslash S$. Finally, let $x \in W \backslash S$. Then $x^{*}=x^{-1} h$, and $x * y * x^{*}=x *\left(y x^{-1} h\right)=x y x^{-1} h$ for $y \in U \backslash S$ and $x * y * x^{*}=(x y) *\left(x^{-1} h\right)=x y x^{-1} h$ for $y \in V \backslash S$.

Proposition 2.4. Consider a group $G=G(\cdot)$. If $G(*)=G[S, h]$, then a (normal) subgroup $H$ of $G(\cdot)$ is a (normal) subgroup of $G(*)$ if and only if $H \leq S$ or $h \in H$.

If $G(*)=G[U, V, h]$, then a subgroup $H$ of $G(\cdot)$ is a subgroup of $G(*)$ if and only if $H \leq U \cup V$ or $h \in H$. The normality is retained if and only if $H \leq S$ or $h \in H$.

Proof. It is clear from the definition of $G(*)$ that a subgroup $H$ of $G(\cdot)$ is a subgroup of $G(*)$, if one of the named conditions holds. From Proposition 2.3 we also see that if $H$ is normal in $G(\cdot)$, and $H \leq S$ or $h \in H$, then it is normal in $G(*)$. When $H \leq G(\cdot)$ does not fulfil $H \leq S$ or $H \subseteq U \cup V$ (the latter means, in fact, $H \leq U$ or $H \leq V$ ), 
then one can find $x \in H$ with $x \cdot x \neq x * x$, and therefore $H$ constitutes in such a case a subgroup of $G(*)$ only when $h \in H$. If $H$ is normal in both $G(\cdot)$ and $G(*)$, and $H$ intersects $U \backslash V$ or $V \backslash U$, then $h \in H$, by Proposition 2.3.

Proposition 2.3 also yields a description of commutators.

Proposition 2.5. If $G(*)=G[S, h]$, then $x y x^{-1} y^{-1}=x * y * x^{*} * y^{*}$ for all $x, y \in$ $G$. Assume $G(*)=G[U, V, h]$, and put $a=x y x^{-1} y^{-1}$ and $b=x * y * x^{*} * y^{*}$, for some $x, y \in G$. Then

$$
b= \begin{cases}a, & \text { if }\{x, y\} \subseteq U \text { or }\{x, y\} \subseteq V \text { or }\{x, y\} \subseteq W \\ a h^{-1}, & \text { if } y \in V \backslash S \text { and } x \notin V, \text { or } x \in W \backslash S \text { and } y \notin W \\ a h, & \text { if } y \in W \backslash S \text { and } x \notin W, \text { or } x \in V \backslash S \text { and } y \notin V .\end{cases}
$$

Proof. If $G(*)=G[S, h]$, then $x y x^{-1}=x * y * x^{*}$, and the commutator equality is clear if $y \in S$. If $y \in G \backslash S$, then $y^{*}=y^{-1} h^{-1}$, and hence $\left(x y x^{-1}\right) * y^{*}=x y x^{-1} y^{*} h=$ $x y x^{-1} y^{-1}$. Assume $G(*)=G[U, V, h]$ and consider the commutators $a$ and $b$. Groups $U$ and $V$ are the same in both operations, and hence $a=b$ if $x, y \in U$ or $x, y \in V$. For $x, y \in W$ use the first part of the proof. Now, if $x \in V \backslash S$ and $y \in U \backslash S$, then $b=\left(x y x^{-1} h^{-1}\right) * y^{-1}=x y x^{-1} h^{-1} y^{-1}=a h$, as required. In the other remaining cases $b=\left(x y x^{-1} h\right) * y^{*}$, and for $y \notin W$ we get $b=x y x^{-1} h y^{-1}$ immediately, while for $y \in W$ the equality $b=x y x^{-1} h y^{-1}$ follows from $b=x y x^{-1} h y^{*} h^{-1}$ and $y^{*}=y^{-1} h$. In all these cases we therefore have $b=a h$ when $y \in W \backslash S$, and $b=a h^{-1}$ when $y \notin W$.

We shall now be concerned with subgroups $\gamma_{i}(G)$ and $\gamma_{i}(G(*))$ that occur in the lower central series, and partly also with the iterated centres $\vartheta_{i}(G)$ and $\vartheta_{i}(G(*))$.

Proposition 2.6. Assume $G(*)=G[S, h]$. Then $\gamma_{i}(G)=\gamma_{i}(G(*))$ for all $i \geq 1$, and $\vartheta_{j}(G)=\vartheta_{j}(G(*))$ for all $j \geq 0$.

Proof. We have $\gamma_{1}(G(\cdot))=G=\gamma_{1}(G(*))$, and $\gamma_{i}(G) \leq S$ for $i \geq 2$. Thus $\gamma_{i}(G)$ is a subgroup of $G(*)$ for $i \geq 2$, and it equals $\gamma_{i}(G(*))$ because of the coinciding commutators. Similarly $h \in \vartheta_{j}(G)$ for $j \geq 1$, and hence $\vartheta_{j}(G)$ is always a subgroup of $G(*)$, by Proposition 2.4. The coinciding conjugation implies $\vartheta_{j}(G)=\vartheta_{j}(G(*))$.

Proposition 2.7. Assume $G(*)=[U, V, h]$. If one of the groups $G(*)$ or $G(\cdot)$ is nilpotent, then both of them are nilpotent, and their nilpotency classes differ at most by one.

Proof. Put $S_{1}=S_{1}^{*}=S$ and define $S_{i+1}\left(\right.$ or $\left.S_{i+1}^{*}\right), i \geq 1$, to be the least subgroup of $G(\cdot)$ (or $G(*))$ that contains all elements $x y x^{-1} y^{-1}$ (or $x * y * x^{*} * y^{*}$ ) with $y \in G$ and $x \in S_{i}$ (or $x \in S_{i}^{*}$ ). Thus $S_{i+1}=\left[S_{i}, G\right]$ in standard notation, and $S_{i+1}^{*}$ is defined in the same way with respect to $G(*)$. If $y \in G$ and $x \in S_{i} \leq S$, then $x y x^{-1} y^{-1}=$ $x * y * x^{*} * y^{*}$, by Proposition 2.5. Hence $S_{i}=S_{i}^{*}$ for all $i \geq 1$. Groups $G(\cdot)$ and $G(*)$ are nilpotent if and only if $S_{k}=1$ for some $k \geq 1$. If this happens and $k$ is minimal, then $1=S_{k} \triangleleft S_{k-1} \triangleleft \ldots \triangleleft S_{1} \triangleleft G$ is a central series of length $k+1$ in both $G(\cdot)$ and $G(*)$. We have $[S, G] \leq[G, G] \leq S$, and $S_{i+1} \leq \gamma_{i+1}(G) \leq S_{i}$ follows by induction, $1 \leq i<k$. Such a result is also true for the operation $*$, and so the length of each of the lower central series is either $k$ or $k+1$. 
Proposition 2.8. Assume $G(*)=G[U, V, h]$. Then $(G(*))^{\prime} \subseteq G^{\prime}$ if and only if $\in G^{\prime}$. When $h \in G^{\prime}$ and $G$ is nilpotent, then the nilpotency class of $G(*)$ does not exceed the nilpotency class of $G(\cdot)$.

Proof. Let $a$ and $b$ be as in Proposition 2.5. If $h \in G^{\prime}$, then $a \in G^{\prime}$ implies $b \in G^{\prime}$, and $(G(*))^{\prime} \leq G^{\prime} \leq S$. If $a$ and $b$ are such that $a \neq b$, then $b \in G^{\prime}$ immediately yields $h \in G^{\prime}$. The rest follows from $G^{\prime} \leq S$.

Proposition 2.9. Assume $G(*)=G[U, V, h]$. The following are equivalent:

(i) $\gamma_{i}(G)$ and $\gamma_{i}(G(*))$ coincide as groups for all $i \geq 2$;

(ii) $G^{\prime}=(G(*))^{\prime}$; and

(iii) $h \in G^{\prime} \cap(G(*))^{\prime}$.

Proof. Points (i) and (ii) are equivalent, since both $G^{\prime}$ and $(G(*))^{\prime}$ always are subgroups of $S$, and $x y x^{-1} y^{-1}=x * y * x^{*} * y^{*}$ when $x \in S$, by Proposition 2.5. That statement also yields the implication (iii) $\Rightarrow$ (ii), while the converse follows from Proposition 2.8.

We conclude this section by a statement which has an obvious proof, but still seems to be worth recording.

Proposition 2.10. Assume $G(*)=G[S, h]$ or $G(*)=G[U, V, h]$. Then $\langle h\rangle$ is a normal subgroup of both $G(\cdot)$ and $G(*)$, and $x\langle h\rangle \mapsto x *\langle h\rangle$ is an isomorphism $G /\langle h\rangle \cong$ $G(*) /\langle h\rangle$.

3. Isomorphisms of related subgroups. Groups $G_{1}$ and $G_{2}$ are said to be 2-related, if $G_{1} \cong G(\cdot)$ and $G_{2} \cong G[S, h]$ for some group $G$. The relation is reflexive (take $h=$ 1) and symmetric (if $G(*)=G[S, h]$, then $G(\cdot)$ can be identified with $G(*)\left[S, h^{-1}\right]$ ). Similarly, we say that $G_{1}$ and $G_{2}$ are 4-related, if there exists $G$ with $G_{1} \cong G(\cdot)$ and $G_{2} \cong G[U, V, h]$. One gets again a reflexive and symmetric relation.

We say that $G_{1}$ and $G_{2}$ are 2,4-related if they are 2-related or 4-related. We refer to the transitive closure of these relations, when we call $G_{1}$ and $G_{2}$ transitively 2-related (or transitively 4-related, or transitively 2,4-related).

Proposition 3.1. Let $G_{1}$ and $G_{2}$ be groups with a common subgroup $S,\left|G_{1}: S\right|=$ $2=\left|G_{2}: S\right|$. If there exist $u_{i} \in G_{i} \backslash S, i \in\{1,2\}$, with $s^{u_{1}}=s^{u_{2}}$ for all $s \in S$, then $G_{1}$ and $G_{2}$ are 2-related.

Proof. Define $\varphi: G_{2} \rightarrow G_{1}$ by $\varphi\left(u_{2} s\right)=u_{1} s$ and $\varphi(s)=s$, for all $s \in S$. Then $\varphi\left(s u_{2}\right)=\varphi\left(u_{2} s^{u_{2}}\right)=u_{1} s^{u_{2}}=u_{1} s^{u_{1}}=s u_{1}$. Define $*$ on $G_{1}$ by $x * y=\varphi\left(\varphi^{-1}(x) \varphi^{-1}(y)\right)$. For all $s, t \in S$ we get $s * t=s t, s * t u_{1}=\varphi\left(s t u_{2}\right)=s \cdot t u_{1}$ and $u_{1} s * t=\varphi\left(u_{2} s t\right)=$ $u_{1} s \cdot t$. Hence $G_{1}(*)=G_{1}[S, h]$ for some $h \in S \cap Z\left(G_{1}\right)$, by Theorem 1.2. Since $\varphi^{-1}$ : $G_{1}(*) \rightarrow G_{2}$ is an isomorphism, the result follows.

COROLlARY 3.2. Let $G_{1}$ and $G_{2}$ be groups with a common subgroup $S,\left|G_{1}: S\right|=2=$ $\left|G_{2}: S\right|$. If there exist $u_{i} \in G \backslash S, i \in\{1,2\}$, with $s^{u_{1}}=s^{u_{2}}$ for all $s \in S$, and such that $u_{1}^{2}=u_{2}^{2}$, then $G_{1}$ and $G_{2}$ are isomorphic, and an isomorphism $\varphi: G_{2} \cong G_{1}$ can be defined by $\varphi(s)=s$ and $\varphi\left(s u_{2}\right)=s u_{1}$ for all $s \in S$.

Proof. We have $\varphi: G_{2} \cong G_{1}(*)$, where $*$ is defined as in the proof of Proposition 3.1. The proof will be complete if we show $x * y=x \cdot y$ for all $x, y \in G_{1}$. This is needed just for $x=s u_{1}$ and $y=u_{1} t$, where $s, t \in S$, and $s u_{1} * u_{1} t=\varphi\left(s u_{2} \cdot u_{2} t\right)=s u_{2}^{2} t=s u_{1}^{2} t=$ $s u_{1} \cdot u_{1} t$. 
Proposition 3.3. Assume $S<G,|G: S|=2$ and $h=z^{x} z$, where $z \in Z(S)$ and $x \in$ $G \backslash S$. Define $\alpha: G \rightarrow G$ by $\alpha(s)=s$ and $\alpha(s x)=s x z$, for all $s \in S$. Then $h \in S \cap Z(G)$, and $\alpha: G[S, h] \cong G$ is an isomorphism.

Proof. The element $h$ belongs to $S \cap Z(G)$, by Lemma 1.6. By Proposition 2.3, $x^{*} * s * x=x^{-1} s x=(x z)^{-1} s(x z)$, for all $s \in S$. Furthermore, $x * x=x x z^{x} z=(x z)(x z)$, and hence $\alpha$ is an isomorphism by Corollary 3.2 (with $u_{2}=x$ and $u_{1}=x z$ ).

THEOREM 3.4. Groups $G_{1}$ and $G_{2}$ are 2-related if and only if there exist groups $H_{i} \cong G_{i}$ with $S<H_{i}$ and $\left|H_{i}: S\right|=2$, and elements $u_{i} \in H_{i} \backslash S, i \in\{1,2\}$, such that $s^{u_{1}}=s^{u_{2}}$ for all $s \in S$.

Proof. If such groups and elements exist, then $G_{1}$ and $G_{2}$ are 2-related by Proposition 3.1. If $G_{1}$ and $G_{2}$ are 2-related, then there exists $G=G(\cdot)$ with $G_{2} \cong$ $G(*)=G[S, h]$ and $G_{1} \cong G(\cdot)$. Put $H_{2}=G(*), H_{1}=G(\cdot)$, choose $u_{1}=u_{2} \in G \backslash S$, and use Proposition 2.3.

Proposition 3.1 and Corollary 3.2 correspond to Lemmas 1.4 and 1.5 of [3].

We now turn to 4-related groups.

Proposition 3.5. Let $G_{1}$ and $G_{2}$ be groups with a common subgroup $S,\left|G_{1}: S\right|=4=$ $\left|G_{2}: S\right|$. If there exist $u_{i}, v_{i} \in G_{i} \backslash S, u_{i} S \neq v_{i} S, i \in\{1,2\}$, with $s^{u_{1}}=s^{u_{2}}$ and $s^{v_{1}}=s^{v_{2}}$ for all $s \in S$, and such that $u_{1}^{2}=u_{2}^{2} \in S$ and $v_{1}^{2}=v_{2}^{2} \in S$, then $G_{1}$ and $G_{2}$ are 4-related.

Proof. Define $\varphi: G_{2} \rightarrow G_{1}$ by $\varphi(s)=s, \varphi\left(u_{2} s\right)=u_{1} s, \varphi\left(v_{2} s\right)=v_{1} s$ and $\varphi\left(u_{2} v_{2} s\right)=$ $u_{1} v_{1} s$, for all $s \in S$. Note that $s^{u_{1} v_{1}}=s^{u_{2} v_{2}}$ for all $s \in S$, and hence $\varphi\left(s w_{2}\right)=$ $\varphi\left(w_{2} s^{w_{2}}\right)=w_{1} s^{w_{2}}=w_{1} s^{w_{1}}=s w_{1}$, if $w_{i}$ stands for $u_{i}, v_{i}$ or $u_{i} v_{i}, i \in\{1,2\}$. Conditions $u_{1}^{2}=u_{2}^{2}$ and $v_{1}^{2}=v_{2}^{2}$ imply that $\varphi$ is an isomorphism when restricted to mappings $S \cup S u_{2} \rightarrow S \cup S u_{1}$ and $S \cup S v_{2} \rightarrow S \cup S v_{1}$, by Corollary 3.2. Define * on $G_{1}$ by $x * y=\varphi\left(\varphi^{-1}(x) \varphi^{-1}(y)\right)$, and put $U=S \cup S u_{1}$ and $V=S \cup S v_{1}$. We shall show that $x * y=x y$ whenever $x \in U$ or $y \in V$. The cases $x, y \in U$ and $x, y \in V$ follow from the mentioned partial isomorphisms. Assume $x=u_{1} s$ and $y=w_{1} t$, where $s, t \in S$ and $w_{i}$ stands for $v_{i}$ or $u_{i} v_{i}, i \in\{1,2\}$. Then $x * y=\varphi\left(u_{2} s \cdot w_{2} t\right)=\varphi\left(u_{2} w_{2} s^{w_{2}} t\right)=u_{1} w_{1} s^{w_{1}} t=$ $u_{1} s \cdot w_{1} t=x y$ (the case $w_{1}=v_{1}$ is clear, and $\left.u_{2}\left(u_{2} v_{2}\right)=u_{1}^{2} v_{2}=v_{2}\left(u_{1}^{2}\right)^{v_{1}}\right)$. Similarly, $x * y=\varphi\left(s w_{2} \cdot t v_{2}\right)=s t^{w_{1}^{-1}} w_{1} v_{1}=x y$ when $y=t v_{1}$ and $x=s w_{1}$, where $s, t \in S$ and $w_{i}$ equals $u_{i}$ or $u_{i} v_{i}, i \in\{1,2\}$. The rest follows from Theorem 1.2, since $\varphi^{-1}: G_{1}(*) \rightarrow G_{2}$ is an isomorphism.

COROLlarY 3.6. Let $G_{1}$ and $G_{2}$ be groups with a common subgroup $S,\left|G_{1}: S\right|=4=$ $\left|G_{2}: S\right|$. Suppose that there exist $u_{i}, v_{i} \in G \backslash S, u_{i} S \neq v_{i} S, i \in\{1,2\}$, with $s^{u_{1}}=s^{u_{2}}$ and $s^{v_{1}}=s^{v_{2}}$ for all $s \in S$, and with $u_{1}^{2}=u_{2}^{2} \in S$ and $v_{1}^{2}=v_{2}^{2} \in S$. Then $\left(u_{1} v_{1}\right)^{2}=\left(u_{2} v_{2}\right)^{2}$ if and only if $\left[u_{1}, v_{1}\right]=\left[u_{2}, v_{2}\right]$, and in such a case $G_{1}$ and $G_{2}$ are isomorphic, and an isomorphism $\varphi: G_{2} \rightarrow G_{1}$ can be defined by $\varphi(s)=s, \varphi\left(s u_{2}\right)=s u_{1}, \varphi\left(s v_{2}\right)=s v_{1}$ and $\varphi\left(s u_{2} v_{2}\right)=s u_{1} v_{1}$, for all $s \in S$.

Proof. We have $\left[u_{i}, v_{i}\right]^{u_{i} v_{i}}=\left(v_{i}^{-2} u_{i}^{-2}\right)^{v_{i}^{-1}}\left(u_{i} v_{i}\right)^{2}, i \in\{1,2\}$. Since $\left[u_{i}, v_{i}\right] \in S$ and $s^{u_{1} v_{1}}=s^{u_{2} v_{2}}$ for all $s \in S$, and since $\left(v_{1}^{-2} u_{1}^{-2}\right)^{v_{1}^{-1}}=\left(v_{2}^{-2} u_{2}^{-2}\right)^{v_{2}^{-1}}$, there is $\left[u_{1}, v_{1}\right]=\left[u_{2}, v_{2}\right]$ if and only if $\left(u_{1} v_{1}\right)^{2}=\left(u_{2} v_{2}\right)^{2}$. Suppose that these equalities hold and note that our definition of $\varphi$ coincides with the definition of $\varphi: G_{2} \cong G_{1}(*)$ in the proof of Proposition 3.5. We shall show that $x * y=x \cdot y$ for all $x, y \in G_{1}$. This is already known for $x \in U=S \cup S u_{1}$ and $y \in V=S \cup S v_{1}$. The mapping $\varphi$ is an isomorphism 
when restricted to $S \cup S u_{2} v_{2} \rightarrow S \cup S u_{1} v_{1}$, by Corollary 3.2, and hence $x * y=x \cdot y$ also holds for $x, y \in W=S \cup S u_{1} v_{1}$.

There are three remaining cases. Consider $s, t \in S$. Assume first $y=u_{1} t \in$ $U \backslash S$. If $x=v_{1} s \in V \backslash S$ then $x * y=\varphi\left(v_{2} s u_{2} t\right)=\varphi\left(v_{2} u_{2} s^{u_{2}} t\right)=\varphi\left(u_{2} v_{2}\left[v_{2}, u_{2}\right] s^{u_{2}} t\right)=$ $u_{1} v_{1}\left[v_{1}, u_{1}\right] s^{u_{1}} t=v_{1} s \cdot u_{1} t=x y$. For the case $x=u_{1} v_{1} s \in W \backslash S$ we get $x * y=$ $\varphi\left(u_{2}^{2} v_{2}\left[v_{2}, u_{2}\right] s^{u_{2}} t\right)=\varphi\left(v_{2}\left(u_{2}^{2}\right)^{v_{2}}\left[v_{2}, u_{2}\right] s^{u_{2}} t\right)=v_{1}\left(u_{1}^{2}\right)^{v_{1}}\left[v_{1}, u_{1}\right] s^{u_{1}} t=x y$.

Finally, let us have $y=u_{1} v_{1} t \in W \backslash S$ and $x=v_{1} s \in V \backslash S$. Then $x * y=$ $\varphi\left(v_{2} u_{2} v_{2} s^{u_{2} v_{2}} t\right)=\varphi\left(u_{2} u_{2}^{-2}\left(u_{2} v_{2}\right)^{2} s^{u_{2} v_{2}} t\right)=u_{1} u_{1}^{-2}\left(u_{1} v_{1}\right)^{2} s^{u_{1} v_{1}} t=x y$, and the proof is complete.

Proposition 3.7. Assume $U<G$ and $V<G$, where $U \neq V$ and $|G: U|=\mid G$ : $V \mid=2$. Consider $u \in U \backslash V, v \in V \backslash U, p \in Q(U \backslash V)$ and $q \in Q(V \backslash U)$, and put $z=$ $p^{v} q$ and $h=z^{u v} z$. Define $\alpha: G \rightarrow G$ by $\alpha(s)=s, \alpha(s u)=\sup , \alpha(s v)=\operatorname{svq}$ and $\alpha(\operatorname{suv})=$ suvz, for all $s \in U \cap V$. Then $h \in Q(U, V)$, and $\alpha: G\left[U, V, h^{-1}\right] \cong G$ is an isomorphism.

Proof. The element $h$ belongs to $Q(U, V)$, by Lemma 1.7. Put $G(*)=G\left[U, V, h^{-1}\right]$. Operations $*$ and c coincide on $U$ and $V$, and hence we need to verify, by Corollary 3.6, just $u * u=u p u p, v * v=v q v q$ and $w * w=w z w z$, where $w=u v$. However, $p u p=u$, as $p \in Q(U \backslash V), q v q=v$, as $q \in Q(V \backslash U)$, and $w * w=w w h=w w z^{w} z=w z w z$.

Note that the definition of $\alpha$ does not depend on the choice of $u \in U \backslash V$ and $v \in V \backslash U$, by Lemma 1.7. Note also that from Lemma 1.4 one sees immediately that the restrictions of $\alpha$ to $U \rightarrow U$ and $V \rightarrow V$ are isomorphisms.

THEOREM 3.8. Groups $G_{1}$ and $G_{2}$ are 4-related if and only if there exist groups $H_{i} \cong$ $G_{i}$ with $S<H_{i}$ and $\left|H_{i}: S\right|=4$, and elements $u_{i}, v_{i} \in H_{i} \backslash S, i \in\{1,2\}$, with $u_{i} S \neq v_{i} S$, $u_{1}^{2}=u_{2}^{2}, v_{1}^{2}=v_{2}^{2}, s^{u_{1}}=s^{u_{2}}$ and $s^{v_{1}}=s^{v_{2}}$, for all $s \in S$.

Proof. Use Proposition 3.5 if there exist groups $H_{i}, i \in\{1,2\}$, and put $H_{2}=G(*)$ and $H_{1}=G(\cdot)$, when $G_{2} \cong G(*)=G[U, V, h]$ and $G_{1} \cong G(\cdot)$.

4. Agents form a vector space. When deciding which groups are 2,4-related to a given group $G=G(\cdot)$, the number of situations to be considered can be often reduced by applying isomorphism statements like Propositions 3.3 and 3.7. One can also use the following lemma (which overlaps with [6, Lemmas 2.4 and 2.5]). The proof of the lemma is easy and hence it is omitted.

Lemma 4.1. Consider a group $G=G(\cdot)$ and an automorphism $\alpha \in \operatorname{Aut}(G)$. Then $\alpha: G[S, h] \cong G[\alpha(S), \alpha(h)]$ and $\alpha: G[U, V, h] \cong G[\alpha(U), \alpha(V), \alpha(h)]$.

Constructions of $G[S, h]$ and $G[U, V, h]$ can be subjected to a superimposition, thus producing an affine behaviour. Propositions 4.2 and 4.3 describe this phenomenon, and we use it below in Propositions 4.4 and 4.5.

Proposition 4.2. Assume $G_{1}=G\left[S, h_{1}\right]$ and $G_{2}=G\left[S, h_{1} h_{2}\right]$, where $h_{1}, h_{2} \in S \cap$ $Z(G)$. Then $G_{2}=G_{1}\left[S, h_{2}\right]$.

Proof. Denote by $*$ the operation of $G_{1}$ and by $\circledast$ the operation of $G_{2}$. Consider $u, v \in G$. If $u \in S$ or $v \in S, u \circledast v=u * v=u v$. If $u, v \in G \backslash S$, then $u \circledast v=u v h_{1} h_{2}=$ $\left(u v h_{1}\right) * h_{2}=u * v * h_{2}$.

Proposition 4.3. Assume $G_{1}=G\left[U, V, h_{1}\right]$ and $G_{2}=G\left[U, V, h_{1} h_{2}\right]$, where $h_{1}, h_{2} \in$ $Q(U, V)$. Then $G_{2}=G_{1}\left[U, V, h_{2}\right]$. 
Proof. Denote by $*$ the operation of $G_{1}$ and by $\circledast$ the operation of $G_{2}$. Consider $x, y \in G$. If $x \in U$ or $y \in V$, then $x \circledast y=x * y=x y$. Assume $x \in G \backslash U$. If $y \in U \backslash V$, then $x \circledast y=x y h_{1} h_{2}=x * y * h_{2}$, and if $y \in G \backslash(U \cup V)$, then $x \circledast y=x y h_{2}^{-1} h_{1}^{-1}=$ $x y h_{1}^{-1} h_{2}^{-1}=x * y * h_{2}^{-1}$.

Proposition 4.4. Assume $S<G$ and $|G: S|=2$. Put $K=\left\{z^{x} z ; z \in Z(S)\right.$ and $x \in$ $G \backslash S\}$. Then $K \leq S \cap Z(G)$, and $(S \cap Z(G)) / K$ is an elementary abelian 2-group. If $h_{1}, h_{2} \in S \cap Z(G)$ and $h_{1} h_{2}^{-1} \in K$, then $G\left[S, h_{1}\right] \cong G\left[S, h_{2}\right]$.

Proof. If $z \in Z(S)$ and $x, y \in G \backslash S$, then $z^{x} z=z^{y} z \in S \cap Z(G)$, by Lemma 1.6. Fix $x \in G \backslash S$. We already know that $K=\left\{z^{x} z ; z \in Z(S)\right\} \subseteq S \cap Z(G)$. Furthermore, $\left(z^{x} z\right)^{-1}=z^{-1}\left(z^{-1}\right)^{x}=\left(z^{-1}\right)^{x} z^{-1}$ and $\left(z_{1}^{x} z_{1}\right)\left(z_{2}^{x} z_{2}\right)=\left(z_{1} z_{2}\right)^{x}\left(z_{1} z_{2}\right)$, for all $z, z_{1}, z_{2} \in$ $Z(S)$. Hence $K \leq S \cap Z(G)$. If $h \in S \cap Z(G)$, then $h^{x} h=h^{2} \in K$, and thus $(S \cap$ $Z(G)) / K$ is an elementary abelian 2-group.

Note now that while $K$ has been defined with respect to the group $G=G(\cdot)$, one gets, by Proposition 2.3, the same subgroup when $G(\cdot)$ is replaced by $G(*)=G[S, h]$, $h \in S \cap Z(G)$. Therefore it suffices to prove $G[S, h] \cong G$ for $h \in K$, by Proposition 4.2. However, such an isomorphism exists by Proposition 3.3.

Proposition 4.5. Let $U<G$ and $V<G$ be subgroups of $G$ with $U \neq V$ and $\mid G$ : $U|=| G: V \mid=2$. Put $S=U \cap V$ and define $W<G$ by $S<W$ and $W / S=U / S$. $V / S$. Furthermore, put $K=\left\{z^{w} z ; z \in Q(U \backslash S) Q(V \backslash S)\right.$ and $\left.w \in W \backslash S\right\}$. Then $K \leq$ $Q(U, V)$ and $Q(U, V) / K$ is an elementary abelian 2-group. If $h_{1}, h_{2} \in Q(U, V)$ and $h_{1} h_{2}^{-1} \in K$, then $G\left[U, V, h_{1}\right] \cong G\left[U, V, h_{2}\right]$.

Proof. Fix $u \in U \backslash S$ and $v \in V \backslash S$. Every $z \in Q(U \backslash S) Q(V \backslash S)$ can be written as $p_{1} q$, where $p_{1} \in Q(U \backslash S)$ and $q \in Q(V \backslash S)$, and $p_{1}$ can be expressed as $p^{v}, p \in$ $Q(U \backslash S)$, because $U^{v}=U$ and $S^{v}=S$. There is $z^{w} z=z^{w^{\prime}} z \in Q(U, V)$ for all $w, w^{\prime} \in$ $W \backslash S$, by Lemma 1.7. Hence $K \subseteq Q(U, V)$ and we can assume $w=u v$. Consider $z, z_{1}, z_{2} \in Q(U \backslash S) Q(V \backslash S)$ and note that they belong to $Z(S)$, by Proposition 1.3. The equalities $\left(z^{w} z\right)^{-1}=z^{-1}\left(z^{-1}\right)^{w}=\left(z^{-1}\right)^{w} z^{-1}$ and $\left(z_{1}^{w} z_{1}\right)\left(z_{2}^{w} z_{2}\right)=\left(z_{1} z_{2}\right)^{w}\left(z_{1} z_{2}\right)$ prove that $K$ is a subgroup of $Q(U, V) . Q(U, V) / K$ is an elementary abelian 2-group, as $h^{2}=h^{w} h \in K$ for every $h \in Q(U, V) \leq Z(W)$.

Group $K$ does not change when $G(*)=G[U, V, h]$ replaces $G(\cdot)$ in the definition of $K$, as $*$ and agree on $U$ and $V, Q^{*}(U, V)=Q(U, V)$, and the conjugation agrees, by Proposition 2.3, when one of the arguments is in $S$. Hence for a proof of $G\left[U, V, h_{1}\right] \cong$ $G\left[U, V, h_{2}\right]$ it suffices to show $G[U, V, h] \cong G$, where $h=z^{w} z \in K$, by Proposition 4.3. However, this follows from Proposition 3.7.

Sets $(S \cap Z(G)) / K$ and $Q(U, V) / K$ are vector spaces over the 2-element field. One also gets a vector space when $K$ is replaced just by $(S \cap Z(G))^{2} \leq K$ or $Q(U, V)^{2} \leq$ $K$, respectively. The corresponding weaker form of Proposition 4.5 appeared in [6 Lemmas 2.6 and 2.7]. Unfortunately, in that paper the proof of its Lemma 2.7 is hard to understand, since it uses, without saying so, a description of isomorphisms corresponding to our Corollary 3.6.

The next statement is presented without a proof, since it is an immediate consequence of Propositions 4.4 and 4.5.

Corollary 4.6. Assume $G(*)=G[S, h]$ or $G(*)=G[U, V, h]$, and suppose that $h$ is of a finite order. Then $G(*) \cong G\left[S, h^{\prime}\right]$ or $G(*) \cong G\left[U, V, h^{\prime}\right]$, respectively, for some $h^{\prime}$ of order a power of two. 
In case of 4-related groups one gets another isomorphism by switching roles of $U$ and $V$. There is $Q(U, V)=Q(V, U)$ and hence for $h \in Q(U, V)$ we can construct both $G[U, V, h]$ and $G\left[V, U, h^{-1}\right]$. Denote the corresponding group operations by $*$ and $\circ$, respectively, and define $\alpha: G \rightarrow G$ by $\alpha(x)=x$ when $x \in U \cup V$ and $\alpha(x)=x h^{-1}$ when $x \in G \backslash(U \cup V)=W \backslash S$. Fix $u \in U$ and $v \in V$. Then $u * u=u^{2}=u \circ u, v * v=v^{2}=$ $v \circ v, u \circ v=u v h^{-1}=(u * v) h^{-1}=\alpha(u * v)$ and $(u \circ v) \circ(u \circ v)=\left(u v h^{-1}\right)\left(u v h^{-1}\right) h=$ $(u v)^{2} h^{-1}=(u * v) *(u * v)$. Hence one can apply Corollary 3.6 to $G_{2}=G(\circ)$, $G_{1}=G(*), u_{1}=u_{2}=u$ and $v_{1}=v_{2}=v$, and we see that $\alpha$ is an isomorphism (as was mentioned in [6]). We can state:

Proposition 4.7. Let $U<G$ and $V<G$ be subgroups of index $2, U \neq V$. Then $G[U, V, h] \cong G\left[V, U, h^{-1}\right]$ for all $h \in Q(U, V)$.

\section{Groups related to finite abelian groups.}

Proposition 5.1. Assume $h \in H \leq G=G(\cdot)$. If $G(*)=G[S, h]$ and $|H: H \cap S|=$ 2, then $H(*)=H[S \cap H, h]$. If $G(*)=G[U, V, h]$ and $|H: H \cap U \cap V|=4$, then $H(*)=H[U \cap H, V \cap H, h]$. If there exists, in addition, $N<G$ such that $N<S$ and $G=H \times N$, then $G(*)=H(*) \times N$, in both cases.

Proof. Since $H \leq G(\cdot)$ contains $h$, it is also a subgroup of $G(*)$, by Proposition 2.4. In case $G(*)=G[S, h]$ we get $h \in H \cap S \cap Z(H)$, and hence the definition of $H(*)$ is just a restriction of the definition of $G(*)$. The same holds in the case $G(*)=$ $G[U, V, h]$, since $h \in Q(U \cap H, V \cap H)$, and $|H: H \cap U \cap V|=4$ implies $H \cap U \cap$ $V=(H \cap U) \cap(H \cap V)<H \cap U<H$ and $H \cap U \cap V<H \cap V<H$. The existence of $N<S$ with $G=H \times N$ means that both $H(*)$ and $N$ are normal in $G(*)$, by Proposition 2.4, and therefore $G(*)=H(*) \times N$ (note that operations $*$ and $\cdot$ coincide on $N)$.

Proposition 5.1 is one of the tools needed to determine groups that are 2-related or 4-related to finite abelian groups. We also need several standard properties of finite abelian groups; the proofs are provided just for the sake of completeness.

LEMMA 5.2. Let $G$ be a finite abelian p-group.

(i) If $g, u \in G$ and $N \leq G$ are such that $G=N \times\langle u\rangle, g \in N$ and $|g| \leq|u|$, then $G=N \times\langle g u\rangle$.

(ii) If $u \in G \backslash G^{p}$, then there exist $N \leq G$ and $g \in G^{p}$ such that $G=N \times\langle g u\rangle$.

(iii) If $h \in G$ is of order $p$, then there exist $N \leq G$ and $u \in G$ such that $G=N \times\langle u\rangle$ and $h \in\langle u\rangle$.

Proof. Assume $G=N \times\langle u\rangle, g \in N$ and $|g| \leq|u|$. If $(u g)^{i} \in N$, then $u^{i} \in N, u^{i}=1$ and $g^{i}=1$. Hence $N \cap\langle g u\rangle=1$, and so $G=N \times\langle g u\rangle$.

Assume $u \in G \backslash G^{p}$ and let $G=C_{1} \times \cdots \times C_{k}$ be a decomposition to cyclic groups. Then $u=u_{1} \ldots u_{k}$ for $u_{i} \in C_{i}, 1 \leq i \leq k$, and we can clearly assume that there exists $r$, $1 \leq r \leq k$, such that $\left|u_{i}\right|=\left|C_{i}\right|$ exactly when $1 \leq i \leq r$, and that $\left|u_{1}\right| \geq \cdots \geq\left|u_{r}\right|$. From point (i) of this lemma we then see that $C_{1}$ can be replaced by $\left\langle u_{1} \ldots u_{r}\right\rangle$, and the rest is easy.

To prove (iii), express $h$ as $h_{1} \ldots h_{k}$, where $h_{i} \in C_{i}, 1 \leq i \leq k$. We can assume that there exists $s, 1<s \leq k$, such that $h_{i} \neq 1$ exactly when $1 \leq i \leq s$, and that $\left|C_{1}\right| \leq \cdots \leq$ $\left|C_{s}\right|$. Let $\left|C_{1}\right|=p^{t}$ and let $u_{i} \in C_{i}, 1 \leq i \leq s$, be such that $\left(\overline{u_{i}}\right)^{p^{t-1}}=h_{i}$. Then one can 
replace $C_{1}$ by $\left\langle u_{1} \ldots u_{s}\right\rangle$, again by point (i) of this lemma, and we can set $u=u_{1} \cdots u_{s}$ and $N=C_{2} \times \cdots \times C_{k}$.

LEMMA 5.3. Let $G$ be a finite p-group, and suppose that $A \times B<G$ is of index $p$. Then there exist $x \in G \backslash(A \times B)$ and $B_{1}, C \leq B$ such that $G=\langle A, x\rangle \times B_{1}, C \leq\langle A, x\rangle$ and $B=C \times B_{1}$, where $C$ is cyclic or trivial.

Proof. Suppose first that there exists $x \in G \backslash(A \times B)$ with $x^{p} \in A$. If $x^{i} a$ lies in $B$, for an integer $i$ and $a \in A$, then $i=p j$ for an integer $j$ since otherwise $x$ would be an element of $A \times B$. But $x^{p j} a \in A$, by our assumption on $x$, and so $x^{i} a=1$. Hence $\langle A, x\rangle \cap B=1$ and $G=\langle A, x\rangle \times B$.

Suppose now $x^{p} \notin A$, for every $x \in G \backslash(A \times B)$. Then $x^{p}=a b$ for some $a \in A$, $b \in B$, and $b \notin B^{p}$. Indeed, if $b$ were equal to some $b_{1}^{p}, b_{1} \in B$, we would have $\left(x b_{1}^{-1}\right)^{p}=$ $a \in A$. Now, $B=B_{1} \times\langle g b\rangle$, for some $g=h^{p}, h \in B$, by point (ii) of Lemma 5.2. Since $x$ can be replaced by $x h$, we see that $g=1$ can be assumed. We have $A \times B=A_{1} \times B_{1}$, where $A_{1}=A \times\langle b\rangle$. Therefore $G=\left\langle A_{1}, x\right\rangle \times B_{1}$, by the first step of this proof, and $\left\langle A_{1}, x\right\rangle=\langle A, b, x\rangle=\left\langle A, x^{p}, x\right\rangle=\langle A, x\rangle$.

The above lemmas will be used just for $p=2$. The latter one remains true when $G /(A \times B)$ is assumed to be cyclic. However, the present form is sufficient for our purposes.

Assume $G(*)=G[S, h]$ or $G(*)=G[U, V, h]$, where $G=G(*)$ is finite abelian. We wish to describe possible isomorphism types of $G(*)$, and hence the order of $h$ can be assumed to be a power of two, by Corollary 4.6. The expression of $G$ as $H \times N$, where $H$ is the Sylow 2-subgroup and $N$ is the subgroup of odd order elements, satisfies the assumptions of Proposition 5.1, and we can therefore consider just the case of $G$ a 2-group.

Let us take $G(*)=G[S, h]$. We are interested only in the cases when $G(*)$ is not isomorphic to $G(\cdot)$, and hence we can assume $h \notin S^{2}$, by Proposition 3.3. Proposition 4.4 yields $G[S, h] \cong G[S, h g]$, for all $g \in S^{2}$, and so, by point (ii) of Lemma 5.2, we can also assume $S=\langle h\rangle \times M$, for some $M \leq G$. Lemma 5.3 then gives $G(\cdot)=\langle h, x\rangle \times N$, for some $x \in G \backslash S$ and $N \leq M$. Put $H=\langle h, x\rangle$, and note that $H /\langle h\rangle$ is cyclic and that $H(*)$ is abelian, by Proposition 2.3. From Proposition 2.10 we see that both $H(\cdot)$ and $H(*)$ are products of at most two cyclic groups. Since $C_{2^{a}} \times C_{2^{b}}$ is 2-related to a $C_{2^{a^{\prime}}} \times C_{2^{b^{\prime}}}$ if and only if these groups have an isomorphic subgroup of index 2, by Theorem 3.4, we conclude that these groups are 2-related if and only $\left(a^{\prime}, b^{\prime}\right)$ or $\left(b^{\prime}, a^{\prime}\right)$ is equal to $(a+1, b-1)$ or $(a-1, b+1)$. We can state:

THEOREM 5.4. Let $G_{1}$ and $G_{2}$ be two finite non-isomorphic abelian groups. Then $G_{1}$ and $G_{2}$ are 2-related if and only if there exist integers $a, b \geq 0, a \neq b$, and an abelian group $N$ such that

$$
G_{1} \cong C_{2^{a+1}} \times C_{2^{b}} \times N \quad \text { and } \quad G_{2} \cong C_{2^{a}} \times C_{2^{b+1}} \times N
$$

\section{COROLlaRY 5.5. All finite abelian 2-groups are transitively 2-related.}

Let us now turn to the situation when $G(*)=G[U, V, h]$ and $G(\cdot) \not G(*)$ is a finite abelian 2-group. Then $h$ has to be an involution, since it belongs to $Q(U, V)$.

Point (iii) of Lemma 5.2 yields the existence of $w \in S$ and $M<S$, for which $h \in\langle w\rangle$ and $S=\langle w\rangle \times M$. A double application of Lemma 5.3 allows us to express $G$ as $\langle x, y, w\rangle \times N$, where $N<S$. Proposition 5.1 makes it possible to treat just the 
group $H=\langle x, y, w\rangle$, and we shall consider separately cases of rank 2 and rank 3 . The case of rank 2 is covered by Proposition 5.6.

Assume $H=\langle x, y, w\rangle=\left\langle u_{1}\right\rangle \times\left\langle u_{2}\right\rangle \times\left\langle u_{3}\right\rangle$. Since $H / H \cap S \cong G / S$ is a 4-group, there exists a nontrivial product of a subset of $\left\{u_{1}, u_{2}, u_{3}\right\}$ that falls into $S$. This product can replace one of its factors in the decomposition of $H$, by point (i) of Lemma 5.2. Thus we can assume $u_{3} \in S$. Consider now $v=v_{1} v_{2} v_{3} \in H$, where $v_{i} \in\left\langle u_{i}\right\rangle, 1 \leq i \leq 3$, are such that $h \in\langle v\rangle,\left|v_{i}\right|=|v|$ or $v_{i}=1$, for all $i$ with $1 \leq i \leq 3$, and $|v|$ is equal to the least $\left|u_{j}\right|$, for which $1 \leq j \leq 3$ and $v_{j} \neq 1$.

If $|v|=\left|u_{1}\right|$, then $H=\left(\langle v\rangle \times\left\langle u_{2}\right\rangle\right) \times\left\langle u_{3}\right\rangle$, by point (i) of Lemma 5.2 (put $N=$ $\left\langle u_{2}\right\rangle \times\left\langle u_{3}\right\rangle, u=u_{1}$ and $\left.g=v u^{-1}\right)$, and we are in the situation of rank 2 again, as $u_{3} \in S$ means that Proposition 5.1 can be applied once more.

Hence we can assume both $|v|<\left|u_{1}\right|$ and $|v|<\left|u_{2}\right|$, which implies $v \in$ $\left\langle u_{1}^{2}, u_{2}^{2}, u_{3}\right\rangle=H \cap S$ and $\left|u_{3}\right|=\left|v_{3}\right|=|v|$. Thus $H=\left\langle u_{1}\right\rangle \times\left\langle u_{2}\right\rangle \times\langle v\rangle, h \in\langle v\rangle$, and one can use Proposition 5.7.

Proposition 5.6. Suppose $H(*)=H[U, V, h]$, where $H=\langle x\rangle \times\langle y\rangle,|x|=2^{a},|y|=$ $2^{b}$ and $a \geq b \geq 1$. Then exactly one of the following cases takes place:

(i) $a=2, b=1$ and $H(*) \cong Q_{8}$;

(ii) $a \geq 2, b=1$ and $H(*) \cong \operatorname{Mod}_{2^{a+1}}$;

(iii) $a \geq 2, \quad b=2$ and $H(*) \cong\left\langle x, y, z ; \quad x^{2^{a}}=y^{2}=z^{2}=1, \quad y z y^{-1}=x z x^{-1}=\right.$ $\left.z, x y x^{-1}=y z\right\rangle$ is a semidirect product of $C_{2} \times C_{2}$ and $C_{2^{a}}$;

(iv) $a \geq 2, b \geq 2$ and $H(*)$ is a semidirect product of two cyclic groups, where $H(*) \cong\left\langle x, y ; x^{2^{a}}=y^{2^{b}}=1, \quad x y x^{-1}=y^{1+2^{b-1}}\right\rangle$ or $H(*) \cong\left\langle x, y ; x^{2^{a}}=y^{2^{b}}=\right.$ $\left.1, y x y^{-1}=x^{1+2^{a-1}}\right\rangle$.

Proof. The only choice for $S$ is $\left\langle x^{2}\right\rangle \times\left\langle y^{2}\right\rangle$, and the subgroups $U, V$ and $W$ match the subgroups $\left\langle x, y^{2}\right\rangle,\left\langle y, x^{2}\right\rangle$ and $\left\langle x y, x^{2}\right\rangle=\left\langle x y, y^{2}\right\rangle$ in some order. Since $H$ can be expressed also as $\langle x y\rangle \times\langle y\rangle$ (by point (i) of Lemma 5.2) we can assume that either $W=\left\langle y, x^{2}\right\rangle$ or $W=\left\langle x y, y^{2}\right\rangle$, with no loss of generality. The roles of $U$ and $V$ are interchangeable, by Proposition 4.7, and hence we also can assume $U=\left\langle x, y^{2}\right\rangle$. Thus the order of $x$ in $H(*)$ always equals $2^{a}$.

Suppose first $b=1$. Then $S=\left\langle x^{2}\right\rangle, h=x^{2^{a-1}}$ and $y * x * y^{*}=x h=x^{1+2^{a-1}}$, by Proposition 2.3, and so $H(*) \cong \operatorname{Mod}_{2^{a+1}}$ if $y * y=1$. Now, $y * y=1$ when $V=\left\langle y, x^{2}\right\rangle$, while in the case $W=\left\langle y, x^{2}\right\rangle$ and $a \geq 3$ we get $y^{\prime}=y x^{2^{a-2}} \in W \backslash S, y^{\prime} * x *\left(y^{\prime}\right)^{*}=$ $y * x * y^{*}$ and $y^{\prime} * y^{\prime}=y^{2} x^{2^{a-1}} h^{-1}=1$. The case $W=\left\langle y, x^{2}\right\rangle$ and $a=2$ yields $y * y=$ $y^{2} h=h$, which means that both $x$ and $y$ are of order 4 in $H(*)$, and therefore $H(*) \cong Q_{8}$.

Assume now $b \geq 2$. Then $h \in\left\{x^{2^{a-1}}, y^{2^{b-1}}, x^{2^{a-1}} y^{2^{b-1}}\right\}$. Consider the automorphism $\alpha$ of $H(\cdot)$ that sends $x$ to $x$ and $y$ to $y x^{2^{a-b}}$. (The existence of the automorphism $\alpha$ follows from point (i) of Lemma 5.2.) Then $\alpha(U)=U$ and $\alpha\left(y^{2^{b-1}}\right)=x^{2^{a-1}} y^{2^{b-1}}$, which means that the case $h=x^{2^{a-1}} y^{2^{b-1}}$ needs not be considered, by Lemma 4.1. We always have $y * x * y^{*}=x h$ and $x * y * x^{*}=y h$, and hence one of the situations described in point (iv) appears when the order of $y$ in $G(*)$ equals $2^{b}$. This is certainly true when $V=\left\langle y, x^{2}\right\rangle$. Let us take $W=\left\langle y, x^{2}\right\rangle$. Then $y * y=y^{2} h$, and hence the order of $y$ in $G(*)$ is $2^{b}$ if $h=x^{2^{a-1}}$ or $b \geq 3$. Assume $b=2$ and $y^{2}=h$. Then $y$ becomes an involution in $G(*)$, and by setting $z=h$ we get the defining relations of (iii).

Proposition 5.7. Suppose $H(*)=H[U, V, h]$, where $H=\langle x\rangle \times\langle y\rangle \times\langle g\rangle,|x|=2^{a}$, $|y|=2^{b}, a \geq b \geq 1,|g|=2^{c}, h=g^{2^{c-1}}$ and $U \cap V=\left\langle x^{2}, y^{2}, g\right\rangle$. Then either

(i) $G(*) \cong\left\langle x, y, z ; x^{2^{a}}=y^{2^{b}}=z^{2^{c}}=1, x z x^{-1}=y z y^{-1}=z, x y x^{-1}=y z^{2^{c-1}}\right\rangle$ is a semidirect product of $C_{2^{b}} \times C_{2^{c}}$ and $C_{2^{a}}$; or 
(ii) $G(*) \cong\left\langle x, y ; x^{2^{a}}=1, y^{4}=1, x y x^{-1}=y^{-1}\right\rangle$ is a semidirect product of $C_{4}$ and $C_{2^{a}}$, and $b=c=1$.

Proof. Groups $U, V, W$ match groups $\langle x, S\rangle,\langle y, S\rangle$ and $\langle x y, S\rangle$ in some order. One can exchange $x$ with $x y$ (by point (i) of Lemma 5.2), and, independently, $U$ with $V$. Hence $U=\langle x, S\rangle=\left\langle x, y^{2}, g\right\rangle$ can be assumed.

From Proposition 2.3 we get $x * g * x^{*}=g, y * g * y^{*}=g$, and the orders of $x$ and $g$ in $G(*)$ are $2^{a}$ and $2^{c}$, respectively. If $y \in W$, then $y * y=y^{2} h^{-1}$, and we see that the order of $y$ is equal to $2^{b}$ if $b \geq 2$ or $y \in V$. But then the defining relations of (i) apply, as $x * y * x^{*}=y h$ holds, by Proposition 2.3 , in every case.

Let us take $W=\langle y, S\rangle=\left\langle y, x^{2}, g\right\rangle$ and $b=1$. If $c \geq 2$, then one can set $y^{\prime}=y g^{2^{c-2}}$ and from $y^{\prime} * y^{\prime}=y^{2} g^{2^{c-1}} h^{-1}=1$ we easily derive that the defining relations of (i) can be used in this case as well.

Suppose, finally, $W=\langle y, S\rangle$ and $b=c=1$. Then $y * y=g=h, y$ is of order 4 and $y^{*}=y h$.

TheOrem 5.8. Let $G=G(\cdot)$ be a finite abelian group and let $G(*)=G[U, V, h]$, where $U, V<G$ are of index $2, U \neq V$, and $h \in Q(U, V)$. Suppose that $G(*)$ is not isomorphic to $G(\cdot)$. Then there exists $N<U \cap V$ such that $G(\cdot)=H(\cdot) \times N$ and $G(*)=$ $H(*) \times N$, where $H(*)$ and $H(\cdot)$ correspond to one of the cases that are described in Propositions 5.6 and 5.7.

We have described the neighbourhood of finite abelian groups with respect to 2related and 4-related groups. In [6] one can find the description of such neighbourhoods for groups $D_{2^{n}}, Q_{2^{n}}$ and $S D_{2^{n}}$ (the latter group is considered just for $n \geq 4$, while the former ones for $n \geq 3$ ). These groups are mutually 2-related, and no other group is 2-related to any of them. Groups $D_{2^{n}}$ and $Q_{2^{n}}$ are 4-related to $S D_{2^{n}}$, and these groups are also 4-related to $D_{2^{n-1}} \times C_{2}$ and $Q_{2^{n-1}} \times C_{2}$, respectively (where $D_{4}=Q_{4}=C_{4}$ ). The only other case when a group is 4-related to $D_{2^{n}}, Q_{2^{n}}$ or $S D_{2^{n}}$ concerns $S D_{2^{n}}$, which is 4-related to a semidirect product of $C_{2^{n-2}} \times C_{2}$ and $C_{2}$.

The theory presented in this paper helped us to establish that any two 2-groups of order 16 are transitively 2,4-related. For order 32 one gets a slightly different situation. By using GAP we found an exceptional group of order 32, which is 2,4-related to no non-isomorphic group. All other groups of order 32 are transitively 2,4-related. The exceptional group (a semidirect product of $C_{4} \times C_{2}$ and $C_{2}$ ) is presented in [6] including the proof of its isolation. A report about the results of computations on 2groups of order $\leq 64$ will appear separately later, and will describe all instances when two corresponding groups are 2-related or 4-related.

We do not know how to decide effectively if two finite 2-groups $G_{1}$ and $G_{2}$ are transitively 2,4-related. However, there are some results in this direction, one of which states that $G_{1}$ and $G_{2}$ are transitively 2,4-related when both of them possess an elementary subgroup of index 2 .

The constructions of $G[S, h]$ and $G[U, V, h]$ can be generalized to constructions, in which $S=U \cap V$ is a normal subgroup of $G, h \in S \cap Z(G)$ or $h \in Q(U, V)$, and $G / S$ is a 2-group that is cyclic or dihedral, respectively. These constructions are described in [5], and an instance when $G / S$ is cyclic and of order 4 connects the exceptional group of order 32 to the other groups of this order.

At this moment we do not know the properties of the generalized constructions in the extent to which this paper covers the basic constructions - this is one of tasks for the future. The aim of [5] has not been to look for such properties, but to show that 
an instance of these generalized constructions is always induced by a situation when, for finite groups $G(\circ)$ and $G(*), n=|G|$ and $d(\circ, *)=n^{2} / 4$, one can find subgroups $X<Y \leq G(\circ),|Y: X|=2$, where for every left coset $\alpha$ of $Y$ and every right coset $\beta$ of $Y$ there exists a left coset $\alpha_{0} \subseteq \alpha$ of $X$ and a right coset $\beta_{0} \subseteq \beta$ of $X$ such that for every $(x, y) \in \alpha \times \beta$ one has $x \circ y \neq x * y$ if and only if $(x, y) \in \alpha_{0} \times \beta_{0}$.

This and other results give us hope that all methods to construct $G(*)$ and $G(\circ)$ with $d(*, \circ)=n^{2} / 4$ will be understood in future.

ACKNOWLEDGEMENT. We thank to the referee for a careful reading of the article, which helped us to remove a number of minor mistakes and inaccuracies.

\section{REFERENCES}

1. D. Donovan, S. Oates-Williams and C. E. Praeger, On the distance between distinct group Latin squares, J. Combin. Des. 5 (1997), 235-248.

2. A. Drápal, How far apart can the group multiplication table be?, European J. Combin. 13 (1992), 335-343.

3. A. Drápal, Non-isomorphic 2-groups coincide at most in three quarters of their multiplication tables, European J. Combin. 21 (2000) 301-321.

4. A. Drápal, On distances of multiplication tables of groups, in Proceedings of Groups St. Andrews 1997 in Bath, C. M. Campbell and E. F. Robertson (eds.) (Cambridge University Press, 1999), 248-252.

5. A. Drápal, On groups that differ in one of four squares, European J. Combin. 23 (2002), 899-918.

6. N. Zhukavets, On small distances of small 2-groups, Comment. Math. Univ. Carolin. 42 (2001), 247-257.

7. N. Zhukavets, Close 2-groups, Ph.D. Thesis (Charles University, Prague, 2002). 